Research Article

\title{
Simulations of Steady Cavitating Flow in a Small Francis Turbine
}

\author{
Ahmed Laouari ${ }^{1}$ and Adel Ghenaiet ${ }^{2}$ \\ ${ }^{1}$ Laboratory of Energetic Mechanics and Engineering (LEMI), Faculty of Engineering, University of Boumerdes, \\ 35000 Boumerdes, Algeria \\ ${ }^{2}$ Laboratory of Energetics and Conversion Systems, Faculty of Mechanical Engineering, \\ University of Sciences and Technology Houari Boumediene, BP32 El-Alia, Bab-Ezzouar, 16111 Algiers, Algeria
}

Correspondence should be addressed to Adel Ghenaiet; ag1964@yahoo.com

Received 30 December 2015; Revised 20 March 2016; Accepted 10 April 2016

Academic Editor: Ryoichi Samuel Amano

Copyright (C) 2016 A. Laouari and A. Ghenaiet. This is an open access article distributed under the Creative Commons Attribution License, which permits unrestricted use, distribution, and reproduction in any medium, provided the original work is properly cited.

\begin{abstract}
The turbulent flow through a small horizontal Francis turbine is solved by means of Ansys-CFX at different operating points, with the determination of the hydrodynamic performance and the best efficiency point. The flow structures at different regimes reveal a large flow eddy in the runner and a swirl in the draft tube. The use of the mixture model for the cavity/liquid two-phase flow allowed studying the influence of cavitation on the hydrodynamic performance and revealed cavitation pockets near the trailing edge of the runner and a cavitation vortex rope in the draft tube. By maintaining a constant dimensionless head and a distributor vane opening while gradually increasing the cavitation number, the output power and efficiency reached a critical point and then had begun to stabilize. The cavitation number corresponding to the safety margin of cavitation is also predicted for this hydraulic turbine.
\end{abstract}

\section{Introduction}

The inward-flow reaction water turbines known as Francis turbines are the result of many years of gradual development, which has resulted in very large units of hydraulic efficiency in excess of $80 \%$ and capable of transforming up to $95 \%$ [1] of the available potential head into electric energy. These machines do not operate exclusively at the best efficiency point but frequently from low to maximum power to meet some requirements. A Francis turbine is usually designed under the cavitation-free condition at the rated load, but unfavorable phenomena affecting its reliability and smooth operation have frequently happened due to the occurrence of cavitation which presents unwanted consequences such as damage to material surfaces and degradation of machine performance. It is difficult to avoid cavitation in hydroturbines which cannot be avoided completely but can be reduced. Computing two-phase cavitating flows is a big challenge since the cavitating bubbles or clouds have very complicated dynamics. In the other side, the tracing of the gas-liquid interface might be possible for a cavitation bubble but impractical for industrial applications. As a result, a local averaging procedure considering a homogeneous liquid-vapor mixture is a reasonable approach as far as the computing time is concerned [2]. The liquid contains microbubbles which under a decreasing pressure may grow and form cavities, and in such a process very large and steep density variations happen in the low-pressure/cavitating regions [3]. Cavitation can occur near the fast moving blades where the local dynamic head increases causing the static pressure to fall. In Francis turbines, the major part of pressure drop occurs in the turbine itself; thus for the design operating range the type of cavity developing in the runner is closely driven by the specific energy coefficient, where high and low values of this coefficient correspond, respectively, to a cavity onset at the leading edge suction side and pressure side of blades, whereas the flow coefficient influences only the cavity whirl [4].

The present day state-of-the-art CFD technique is considered as an alternative tool to provide insight into the flow characteristics of hydropower components, Helmut et al. [5] predicted the hill chart of a high specific speed Francis turbine by using CFX-TASCflow, where the steady state interactions between stationary and rotating components were simulated 
by a mixing plane between the components for several operating points. The comparison between experimentally and numerically evaluated hill charts is impressive and the best efficiency point is identical in both hill charts. Mirjam et al. [6] described a simulation method for a complete hydraulic turbine, from the spiral casing through the distributor and runner to the outlet draft tube and their main conclusion is that the stage interface provides better predictions of flow field for the design and off-design operating points as it takes into account the interactions between all components. The most important ones are the effect of the runner on the wicket gate and vice versa. Sabourin et al. [7] implemented a strategy to simulate flow interactions between rotating and stationary components. The distributor and the runner were calculated in a single calculation through the stage interface, whereas the draft tube was calculated separately and the pressure condition at runner outlet is adjusted. Wu et al. [8] applied CFD to a Francis turbine to integrate three blade designs in order to provide over $3 \%$ increase in peak efficiency and $13 \%$ increase in power with an improved cavitation coefficient for less than 0.09. In comparison with the original runner, they demonstrated that the pressure exhibits a much more uniform distribution without a low-pressure zone on the suction side near the leading edge. Kumar and Saini [9] presented a study for different causes of the declined performance of hydroturbines and the suitable remedial measures based on a literature survey of various aspects related to cavitation. Senocak and Shyy [10] used the mass fraction equation and $k$ - $\varepsilon$ turbulence model as well as the pseudocompressibility method to simulate the $3 \mathrm{D}$ and axisymmetric cavitating flows. The mixture model has been developed from mass transfer expressions, and one is based on the bubble dynamics whereas the others are established from the mechanics of evaporation and condensation. Wu et al. [11] presented the simulation results for the cavitating turbulent flow in a Francis turbine using the mixture model for cavity-liquid two-phase flows. In such a model an improved expression for mass transfer is employed based on evaporation and condensation mechanisms and considering the effects of nondissolved gas, turbulence, tension of interface at cavity, and phase change rate. Liu et al. [12] used the mixture model in the cavitating turbulent flow analysis and showed that the differences between the results of the single phase simulation and those of the cavitating flow are almost negligible due to the high cavitation number at the corresponding operating condition, except for the part load. Furthermore, the cavitated vortex rope is observed in the draft tube at overload condition and its configuration in the inlet cone is visualized using the criterion that the rope boundary is given by the cavity volume fraction of $10 \%$. Kurosawa et al. [13] presented a high accuracy performance prediction method for a Francis turbine using RANS equations combined with Reynolds-stress model and bubble two-phase model with consideration of cavitation but imitated with the modified Rayleigh-Plesset equation. As a result, the main cause of efficiency drop is the increase of draft tube loss and under the critical cavitation condition the volume ratio of cavity is increased sharply; in addition, the prediction results for cavitation in the runner outlet passage are in good agreement with the visualization test results. $\mathrm{Wu}$ et al. [14] applied simulations based on $k$ - $\omega$ SST turbulence model and mixture model as well as a model test to study the cavitation flow in a Francis turbine and to calculate the gradient of equal critical cavitation coefficient line which does not change with the decrease of unit speed and the opening of distributor vanes at the highest efficient region. Yaping et al. [15] used different turbulence models to investigate numerically the performance of a Francis turbine and by comparing with experimental results they found a certain deviation for different turbulence models, and the main causes of big difference between the simulation and the model test include on one hand the mesh generation and boundary conditions and on the other hand the difficulty in simulating accurately the unstable flow such as impact, separation, and vortex. Furthermore, predicted larger circulation with SST turbulence model leads to a higher hydraulic efficiency than with standard $k-\varepsilon$ turbulence model. Yang et al. [16] discussed the spiral cavitating vortex rope developed in the draft tube of a Francis turbine under part load condition, upon simulating the cavitating flow with LES turbulence model and ZGB cavitation model. The results proved the existence of an interaction between the runner cavitation and the spiral vortex rope. The swirl flow at the runner outlet plays an important role in the formation of vortex rope, causing asymmetric cavitation which gives rise to modifications of the swirl flow at the runner blades and thereby affects the characteristics of the vortex rope. Wack and Riedelbauch [17] studied the occurrence of cavitating interblade vortices at deep part load conditions in a Francis turbine using two-phase flow simulations and highlighted the necessity of fine grids in the runner to resolve the vortex core of the interblade vortices. Mousmoulis et al. [18] studied the effects of draft tube vortex cavitation on Francis turbine, numerically and based on the measurements of dynamic pressure and vibration. The results show that the vortex rope frequency has been detected at $25 \%$ and $28 \%$ of the runner speed in the computational and experimental results, respectively, and the intensity of the vortex rope decreases along the draft tube. Gohil and Saini [19] by using Ansys-CFX code investigated the effect of temperature, suction head, and flow velocity on the cavitation of Francis turbine. The discussion of the temperature effect on efficiency loss and cavitation rate used data generated through simulations for three different temperatures and different values of suction head and flow velocity. As a result, the efficiency loss increases with the temperature for all values of suction head and flow velocity, and the cavitation rate increases with the temperature and follows a similar trend to efficiency loss. Decaix et al. [20] investigated by using Ansys-CFX software the vortex rope in a reduced scale model of Francis turbine at full-load condition and two operating points which differ by their cavitation number. The phase change is taken into account through the transport equation of gas volume fraction with a source term based on the simplified Rayleigh-Plesset equation. The global performance depicts an underestimation of the specific energy compared to the experimental results; however, the draft tube wall pressure is well captured. Moreover, at the unstable operating point a qualitative agreement regarding the circumferential velocity field is observed but underestimated at the boundary 
between the liquid and the vapor zones. Kassanos et al. [21] studied numerically the draft tube cavitation performance of a low specific speed Francis turbine and compared the flow field and pressure distributions for different splitter blades and area ratios and showed the benefit of incorporating splitter blades to the runner performance at off-design operation. In a more recent work, Gohil and Saini [22] investigated the performance parameters and cavitating flow in a small Francis turbine by using the code Ansys-CFX. Three different operating conditions with and without cavitation at part load and overload conditions for a plant sigma factor were investigated. They observed that the variation in efficiency as well as the vapor volume fraction is found to be nominal between cavitation and without cavitation conditions.

This paper contributes to predicting the hydraulic performance and cavitating flows in the full passages of a small model of a Francis turbine at different operating conditions, by means of Ansys-CFX solver and considering $k-\omega$ SST turbulence model and the liquid-vapor mixture model. The flow structures and losses in various parts of the turbine were investigated and analyzed at different operating conditions and compared with the results of other investigators for this type of hydraulic turbines. The effects of cavitation model and the setting level on the hydraulic efficiency and the produced power were assessed for several operating conditions and cavitation number.

\section{Computational Domains and CFD Model}

The model of a Francis turbine is taken from a hydraulic laboratory workbench, which includes a spiral case and a distributor with six adjustable vanes to make the right-angle turn and to control and feed the flow to the runner which has ten blades. The draft tube (transparent wall) decelerates the water flow leaving the runner and converts the excess of kinetic energy into a static pressure rise. The obtained CAD model is shown in Figure 1 and the parameters of the turbine are summarized in Table 1.

2.1. Grids Generations. The computational model is separated into four domains meshed separately and connected by interfaces. The distributor vanes and the runner used TurboGrid. Local refinements to boundary layers in the runner were applied to ensure values of $y^{+}$are compatible with the chosen $k$ - $\omega$ SST turbulence model, since this study is focused on understanding the interaction between the components and the associated performance rather than the accurate prediction of vortex structures. Furthermore, this turbulence model, developed by Menter [23], has a good accuracy for boundary layer in adverse pressure gradients and separating flows. Near walls, nodes were positioned in such a way that the value of $y^{+}=\rho y_{p} u_{t} / \mu_{f}, u_{t}=V_{f} \sqrt{f / 2}\left(V_{f}\right.$ : flow velocity), and the friction factor is given by $f=0.025 R_{\text {ex }}^{-0.1428}$.

CFD solution is greatly affected by the size of grid elements, and this is why a grid dependency study was done for five different grid sizes varied from 1.2 to 3.8 million. Accordingly, the hydraulic efficiency revealed a stability for a total grid size equal to 3.2 million. The number of nodes

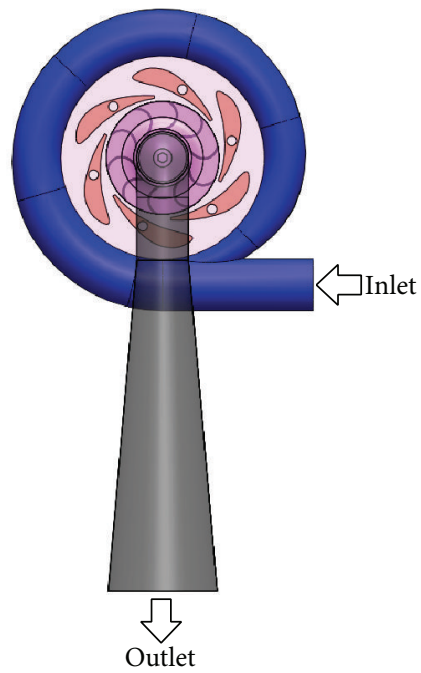

Figure 1: CAD model of Francis turbine.

TABLE 1: Geometrical parameters of Francis turbine model.

\begin{tabular}{lc}
\hline Parameter & $\begin{array}{c}\text { Value } \\
\text { dimension in } \mathrm{mm}\end{array}$ \\
\hline Inlet diameter of spiral case & 38 \\
Outlet diameter of spiral case & 149 \\
Height of distributor vanes & 8 \\
Inlet diameter of runner & 83 \\
Outlet diameter of runner & 38 \\
Outlet diameter of draft tube & 80 \\
Number of blades of guide vane & 6 \\
Number of blades of runner & 10 \\
\hline
\end{tabular}

in each part is as follows: in the distributor vanes it is equal to 870116 , in the runner it is equal to 1689290 , and in the spiral case, meshed by tetrahedral and hexahedral elements, the nodes number is equal to 250354, and for the draft tube it is equal to 390804 nodes. Figure 2 shows the computational meshes of spiral case, distributor vane, runner, and draft tube.

2.2. Boundary Conditions. The simulation was carried out with complete flow passages consisting of spiral casing, distributor vanes, runner blades, and draft tube. The boundary conditions needed in the present simulations are as follows: mass flow set at the casing inlet and a static pressure at the outlet of draft tube. These are widely accepted boundary conditions for the simulation of hydraulic machines $[19,24-$ 26]. On the solid walls of the domain, the nonslipping flow condition was adopted. The interactions between the runner and the distributor vanes and that between the runner and the draft tube are included via the frozen rotor interface. The stage interface is used to predict the hydraulic performance.

2.3. Solver Description. The Ansys-CFX solver [27] which is an element-based finite volume method solves the equations 


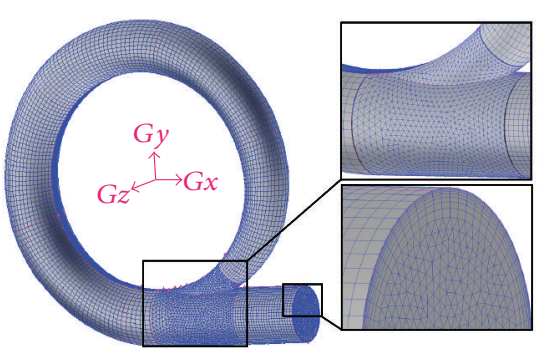

(a) Spiral case

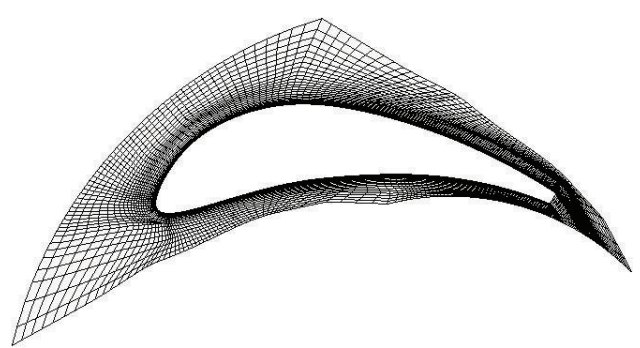

(b) Distributor vane

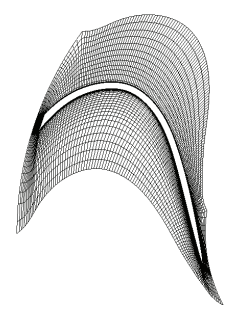

(c) Runner

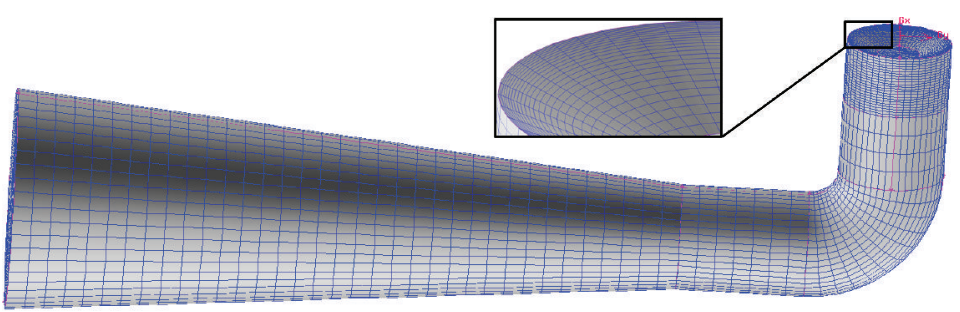

(d) Draft tube

FIGURE 2: Grids of the computational domains.

governing the fluid flow motion represented by the mean form of the Navier-Stokes equations:

$$
\begin{aligned}
& \frac{\partial \rho}{\partial t}+\frac{\partial}{\partial x_{j}}\left(\rho u_{j}\right)=0, \\
& \frac{\partial}{\partial t}\left(\rho u_{i}\right)+\frac{\partial}{\partial x_{j}}\left(\rho u_{j} u_{i}\right)=-\frac{\partial P}{\partial x_{i}} \\
& \quad+\frac{\partial}{\partial x_{j}}\left[\mu_{\mathrm{eff}}\left(\frac{\partial u_{i}}{\partial x_{j}}+\frac{\partial u_{j}}{\partial x_{i}}\right)\right]+S_{u i}, \\
& \vec{S}_{u}=-2 \vec{\Omega} \times \vec{U}-\vec{\Omega} \times(\vec{\Omega} \times \vec{r}), \\
& \frac{\partial}{\partial t}(\rho H)-\frac{\partial P}{\partial t}+\frac{\partial}{\partial x_{j}}\left(\rho u_{j} H\right)=-\frac{\partial}{\partial x_{j}}\left(\lambda \frac{\partial T}{\partial x_{j}}\right. \\
& \left.\quad+\frac{\mu_{t}}{\operatorname{Pr}_{t}} \frac{\partial h}{\partial x_{j}}\right) \\
& \quad+\frac{\partial}{\partial x_{j}}\left\{u_{i}\left[\mu_{\mathrm{eff}}\left(\frac{\partial u_{i}}{\partial x_{j}}+\frac{\partial u_{j}}{\partial x_{i}}\right)-\frac{2}{3} \mu_{\mathrm{eff}} \frac{\partial u_{l}}{\partial x_{l}} \delta_{i j}\right]\right. \\
& \left.\quad+\mu \frac{\partial k}{\partial x_{j}}\right\}+S_{E}, \\
& H+h+\frac{u_{i} u_{i}}{2}+k .
\end{aligned}
$$

The governing equations are integrated over each control volume. The volume's integral is evaluated by considering the flow properties as constant on a control volume and set equal to the central value (mesh node). The surface integrals are evaluated at the integration points which are located at the center of each surface segment of the control volume. The solution field or solution gradients are approximated at the integration points by the nodal values, performed by using finite-elements. The advection scheme in Ansys-CFX is presented in the form of $\phi_{i p}=\phi_{u p}+\beta \nabla \phi \cdot \Delta \sigma$. The high resolution scheme computes $\beta$ locally to be as close to 1 as possible without introducing local oscillations and sets $\nabla \varphi$ equal to the control volume gradient at the upwind node.

2.4. Cavitating Flow Modeling. In the mixture model, it is assumed that there exist the dynamic balance and the diffusion balance in both the liquid phase and the cavity phase of the cavitating flow and the velocities; temperatures and densities of both phases are identical to each other at every position in the whole two-phase flow field. The present used mixture model of cavity/liquid two-phase flow [12] is developed as follows.

Continuity equation of mixture is

$$
\frac{\partial \rho}{\partial t}+\frac{\partial}{\partial x_{j}}\left(\rho u_{j}\right)=0 .
$$

Continuity equation of cavity phase is

$$
\frac{\partial \alpha_{\mathrm{ca}} \rho_{\mathrm{ca}}}{\partial t}+\frac{\partial}{\partial x_{j}}\left(\alpha_{\mathrm{ca}} \rho_{\mathrm{ca}} u_{j}\right)=S .
$$

Momentum equations of mixture are

$$
\frac{\partial\left(\rho u_{j}\right)}{\partial t}+\frac{\partial}{\partial x_{j}}\left(\rho u_{j} u_{i}\right)=\rho g_{i}-\frac{\partial p}{\partial x_{i}}+\frac{\partial \tau_{j i}}{\partial x_{i}} .
$$

It is noted that (3) is the model for calculating the cavitating flow based on evaporation and condensation mechanism attributed to Okita and Kajishima [28], where the static pressure $p$ and vapor pressure $p_{v}$ are replaced with $p^{*}$ and 
$p_{v}^{*}$, respectively, to consider the effects of surface tension, turbulence effect and nondissolved gases:

$$
p^{*}=p+\frac{2 \gamma}{r} \text {. }
$$

Equation (5) for the apparent vapor pressure $p_{v}^{*}$ is introduced to describe the turbulence effect [29] in the vapor pressure $p_{v}$ :

$$
p^{*}=p_{v}+0.195 \rho k
$$

The term $S$ in (3) is adopted to express the mass transfer rate per volume proposed by Cammenga [30].

If $p^{*}>p_{v}^{*}$ (condensation being dominant),

$$
S=\frac{3 \alpha_{\text {ca }}}{r} C_{1}\left(\frac{M}{2 \pi R}\right)^{1 / 2}\left(\frac{p_{v}^{*}}{\sqrt{T}}-\frac{p^{*}}{\sqrt{T}}\right) .
$$

If $p^{*}<p_{v}^{*}$ (evaporation being dominant),

$$
S=\frac{3\left(1-\alpha_{\mathrm{ca}}-\alpha_{u}\right)}{r} C_{2}\left(\frac{M}{2 \pi R}\right)^{1 / 2}\left(\frac{p_{v}^{*}}{\sqrt{T}}-\frac{p^{*}}{\sqrt{T}}\right),
$$

where correction coefficients $C_{1}=0.13$ and $C_{2}=0.01$ [12] are used to consider the effect of phase change rate. By considering the effect of nondissolved gases [19], the density of mixture is expressed as

$$
\rho=\alpha_{\mathrm{ca}} \rho_{\mathrm{ca}}+\alpha_{u} \rho_{u}+\left(1-\alpha_{\mathrm{ca}}-\alpha_{u}\right) \rho_{l} .
$$

$\rho_{\mathrm{ca}}$ is the density of statured vapor and $\rho_{u}$ is the density of nondissolved gases, both of which are assumed as an ideal gas: in the case of water, the initial mass fraction of cavity phase $f_{\mathrm{ca}}=\rho_{\mathrm{ca}} \alpha_{\mathrm{ca}}=1-77 \mathrm{mg} / \mathrm{m}^{3}$ and the initial nuclei radius $r_{0}=3-10 \mu \mathrm{m}$, according to Liu et al. [12], are used to get the number of cavities in a unit volume.

2.5. Simulations Procedure. The present study was performed for the entire flow rate range of this small Francis turbine at a constant head $(H=12 \mathrm{~m})$, for different rotational speeds of the runner $(1100,1500,1900$, and $2360 \mathrm{rpm})$ and for four vanes openings $(32 \%, 64 \%, 72 \%$, and $100 \%)$. The RANS equations were firstly solved without cavitation using the stage interface which performs a circumferential averaging to predict the global turbine performance. On the other side, the frozen rotor interface was considered for the analysis of flow structures through this Francis turbine. This type of an interface produces a steady state solution to multiple frames of reference with some account of interactions between them and is useful when the circumferential variation of the flow is large relative to the component pitch.

With the cavitation model, the suction head and the vapor pressure that depends on temperature are considered with respect to the operating conditions. The solution obtained without cavitation was used as initial result to carry out the computation of cavitating flows.

\section{Results and Discussions}

Firstly, the results of the global hydrodynamic performance parameters characterizing this Francis turbine are presented

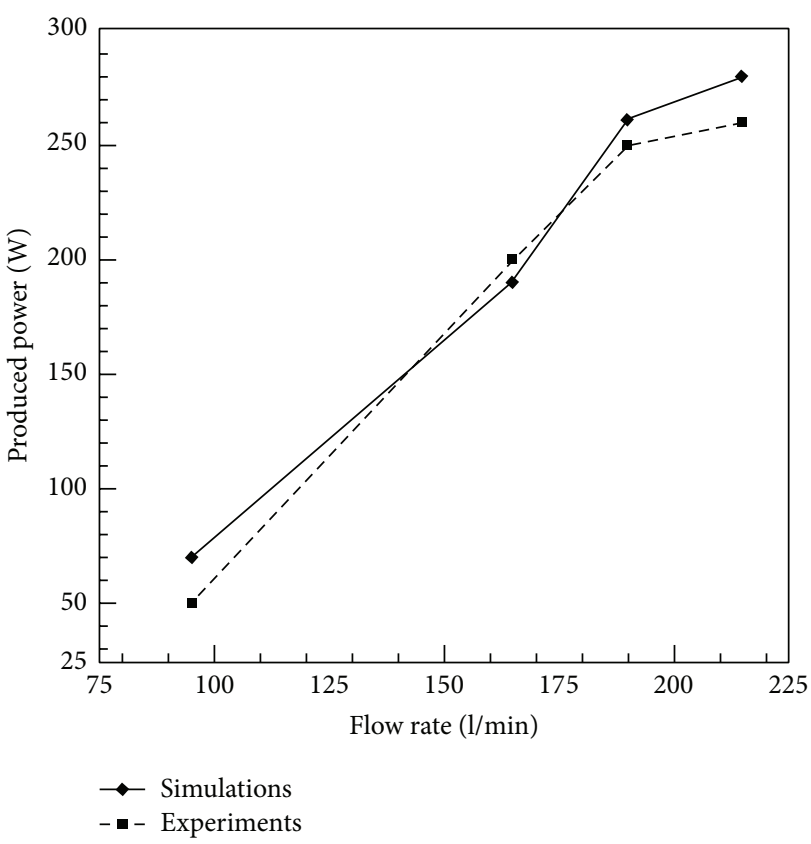

FIgURE 3: Produced power and comparison between prediction and test.

for different rotational speeds of the runner $(1100,1500$, 1900 , and $2360 \mathrm{rpm}$ ) and for four distributor vanes openings (32\%, 64\%, $72 \%$, and $100 \%)$. Secondly, the visualization of internal flows through the turbine components with and without cavitation model is presented. Finally, the hydraulic efficiency and power were calculated based on the data obtained from the simulations without and under cavitation for the purpose of evaluating the performance drop. Figure 5 presents the comparison of the produced power between the CFD prediction and the tests (see Figure 3). It can be readily observed that the accuracy of CFD prediction is satisfactory along the flow rate range.

3.1. Hydrodynamic Performance. The hydraulic efficiency is calculated using the relation defined as follows, where $C$ is the torque produced by the runner: $\eta_{h}=(C \cdot \omega) / \rho g Q H$. In order to compare turbines of different dimensions, dimensionless coefficients in terms of angular speed $\omega$, head $H$, discharge flow rate $Q$ and runner outlet diameter $D$ are used such as unit speed $N_{r}=N D / \sqrt{H}$, unit discharge $Q_{r}=Q / D^{2} \sqrt{H}$, and unit power $P_{r}=P / D^{2} H^{3 / 2}$, in addition to energy coefficient $\psi=8 g H / D^{2} \omega^{2}$ and discharge coefficient $\Phi=$ $8 Q / \pi D^{3} \omega$. The specific speed $\omega_{s}=\Phi^{1 / 2} / \psi^{3 / 4}$ in the case of a Francis turbine is in between 0.14 and 0.65 [31]. The evolutions of the produced power at different rotational speeds and flow rates are illustrated in Figure 4. For all rotational speeds, the produced power increases with the volume flow rate but at different scales. For the operating speed of $2360 \mathrm{rpm}$, the power sweeps the whole operating range for $470 \mathrm{~W}$, whereas at the lowest rotational speed of $1100 \mathrm{rpm}$ the gain in power is $124 \mathrm{~W}$. Figure 5 presents the hydraulic efficiency at different volume flow rates and 


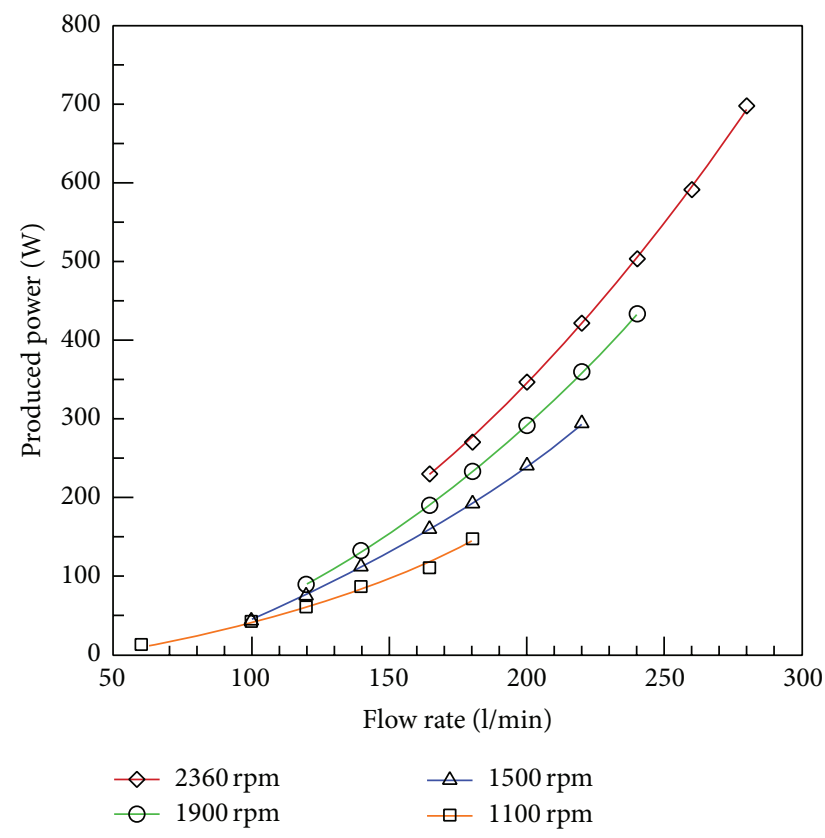

Figure 4: Produced power.

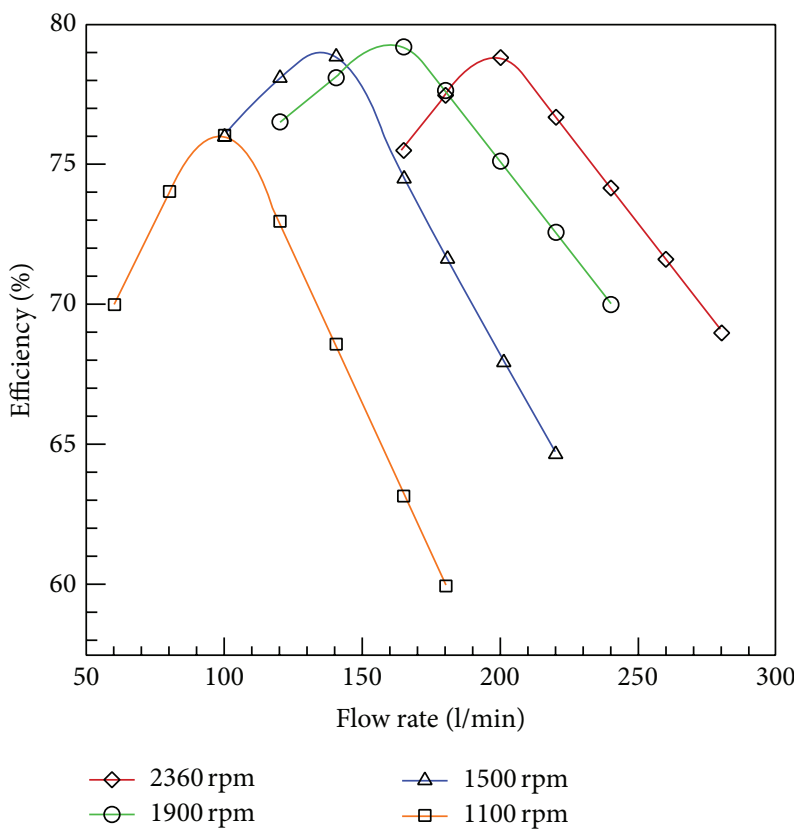

Figure 5: Hydraulic efficiency.

rotational speeds, showing that the performance tendency is similar between all rotational speeds. The point of maximum hydraulic efficiency of $79.18 \%$ is seen at a flow rate of $165 \mathrm{l} / \mathrm{min}$ and a rotational speed of $1900 \mathrm{rpm}$, which is considered as the optimum (nominal) operating point for this Francis turbine. The total pressure head versus the specific speed at the nominal rotational speed and vanes openings reveals that the range of specific speed is between 0.12 and 0.37 , and for the given head it is possible to operate at the best combination between specific speed and vane opening.

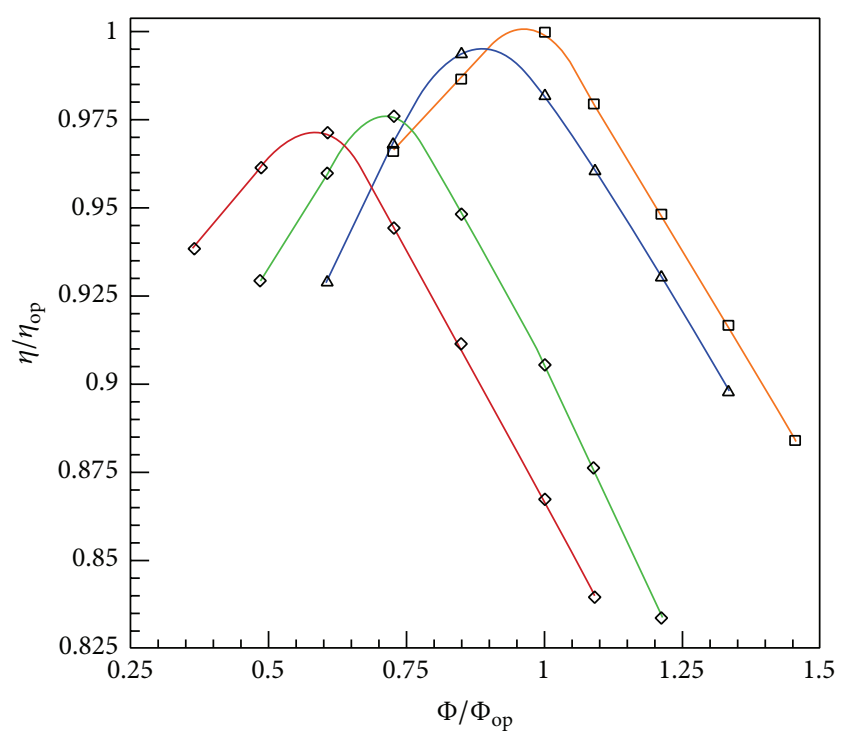

Rotational velocity: $1900 \mathrm{rpm}$

$\begin{array}{ll}\square \text { Opening } 100 \% & \diamond \text { Opening } 54 \% \\ \triangle \text { Opening } 72 \% & \diamond \text { Opening } 32 \%\end{array}$

Figure 6: Ratio of efficiency versus coefficient of discharge.

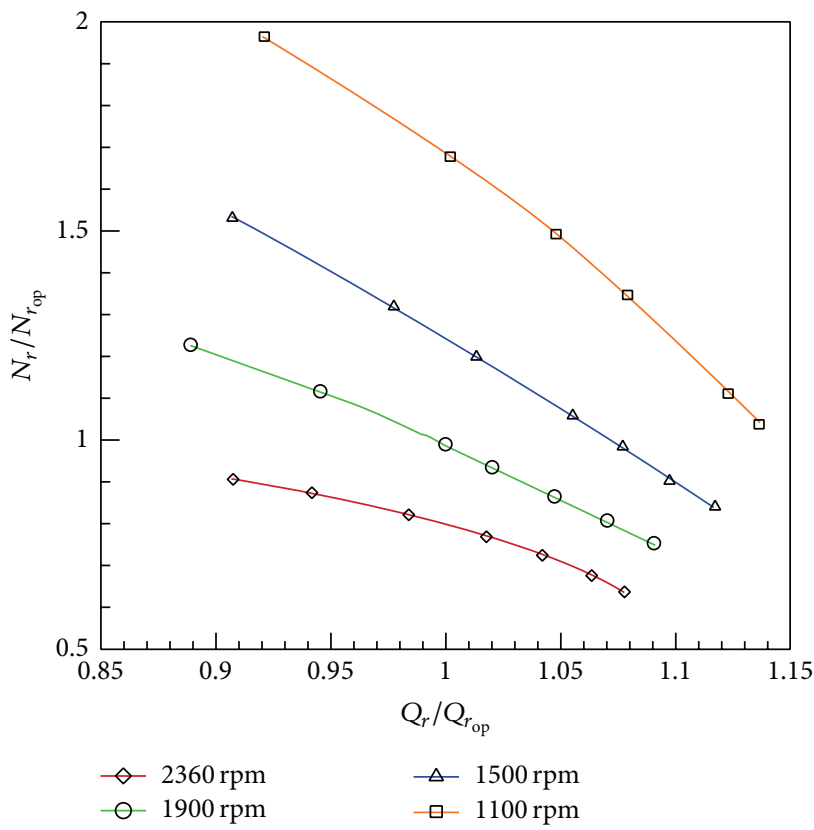

FIgURE 7: Ratio of unit speed versus ratio of unit discharge.

Figure 6 presents the ratio of hydraulic efficiency for different vanes openings at a constant speed, which has a peaked shape. The maximum hydraulic efficiency does not degrade too much with the coefficient of discharge, explaining the large operating range for this turbine and the acceptable efficiency. Figure 7 shows the relationship between the ratio of unit speed and the ratio of unit discharge, revealing a continual decrease at all rotational speeds. For a rotational speed of $1100 \mathrm{rpm}$, the ratio of unit speed sweeps 


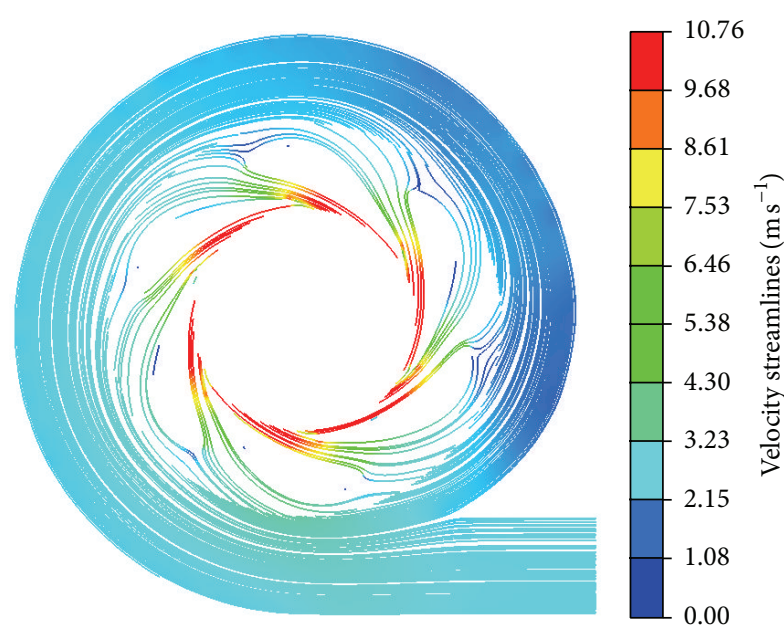

FIGURE 8: Streamlines at midplane of spiral casing and distributor at operating point (BEP).

the whole operation range for a value of 0.9 , whereas for $2360 \mathrm{rpm}$ it is only equal to 0.2 . Further, the unit discharge varies proportionally to the flow rate at the inlet of the spiral casing provided that the total head is constant.

The predicted performance curves are compared with the work of Aggidis and Židonis [32], who presented a fully automated testing procedure of different turbine prototypes and converting the acquired data into hydraulic efficiency hill charts. The small difference between the actual results and the performance curves [32] at the best efficiency point is explained by the fact that the high resolution scheme and the turbulence model performed well. At operating conditions away from the BEP, as explained in $[24,33]$, the turbulence models showed difficulty in correctly capturing the flow features and underestimated losses, thus predicting a higher hydraulic efficiency. Such a difference may be also attributed to the difficulty in capturing the vortex breakdown in the draft tube cone and the losses in the spiral case.

3.2. Flow Structure Analysis. Figure 8 shows the uniform distribution of velocity streamlines in the spiral casing and the distributor for the optimal flow rate. There are no flow collisions at the inlet of distributor vanes and no separations at outlet, conformed to the good setting of vanes at the optimum point (BEP). The stagnation point at the leading edge (LE) is clearly visible where the corresponding value of static pressure is maximum. At the trailing edge of distributor vanes, there is a small flow recirculation in the wake region due to the difference of velocities between the two sides. Furthermore, the angle of distributor vanes as well as its profile is reasonably designed to achieve flow stability and a wide range of maximum hydraulic efficiency. The strong flow acceleration towards the runner the flow quickly covers up the wake regions near the distributor vanes and minimizes it.

The static pressure distribution (Figure 9) is in coherence with the uniform flow distributed evenly around the casing, also seen at small flow rate which reveal the reasonable

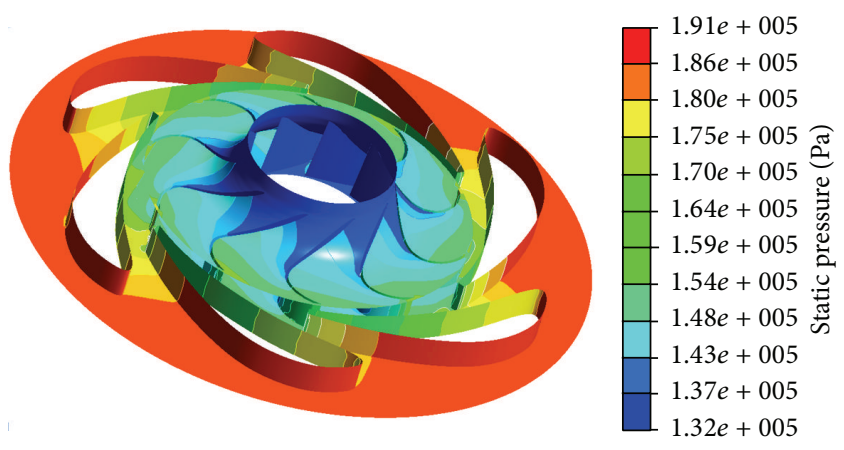

FIGURE 9: Static pressure contours at operating point (BEP) over the vanes and runner blades.

design of the spiral casing to sustain a uniform flow at offdesign operations. The pressure at the distributor vanes is about $191 \mathrm{kPa}$ and decreases to $137 \mathrm{kPa}$ and continues to drop significantly as the water flow advances inward the runner from a radial to an axial direction. The wakes behind the distributor vanes incite important pressure fluctuations within the runner producing irregular torque.

Figure 10 presents the flow velocity contours, for the optimal point (BEP) (flow rate of $165 \mathrm{l} / \mathrm{min}$ ), in the distributor vanes and the runner, showing that the flow gradually transforms into a vortex-free flow as the flow advances inward. The flow velocity in the runner reveals that the flow velocity at the runner entry is not uniform, and there is a noticeable recirculation region over the pressure side characterized by a balance between the centrifugation and the viscous effect equal to the pressure gradient. For all spans, there are recirculation regions over the pressure side from the entry of the runner.

In the interblade channels, the decrease in flow speed near hub and shroud makes the fluid more susceptible to pressure gradient, with a fluid migration from pressure side to suction side leading to radial movements and a passage vortex. As observed from Figure 11(a), there is presence of vorticity regions over the pressure side of some blades for the optimal point (BEP). But for small flow rates such as $951 / \mathrm{min}$ (Figure 11(b)) the vortices occupy all passages and become larger. The morphology of the flow through the runner is greatly affected for the high water flow rates, for example at 215 l/min, where eddies (Figure 11(c)) tend to disappear and the streamlines over the pressure side tend to depart from certain stagnation points.

The incorporated elbow type draft tube with a single outflow channel decelerates the flow leaving the runner and converts the excess of outlet kinetic energy into a static pressure. Figure 12 plots the pressure contours for the optimal BEP, showing the diffusion process which has qualitatively similar trends as reported in $[34,35]$. The pressure near the inner wall is higher due to the channel bend and accordingly a vent set may be chosen. At the bend, a maximum velocity appears near the inner wall and as the flow progresses into the duct and due to inertia force this maximum point rapidly moves towards the outer wall. At the optimal BEP, the flow in the entire draft tube is free of separation. 


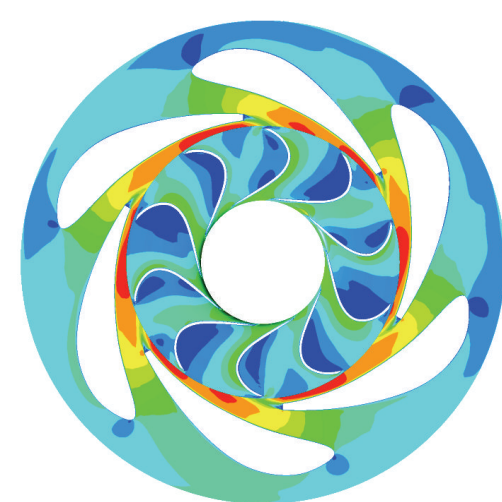

Span: 0.1

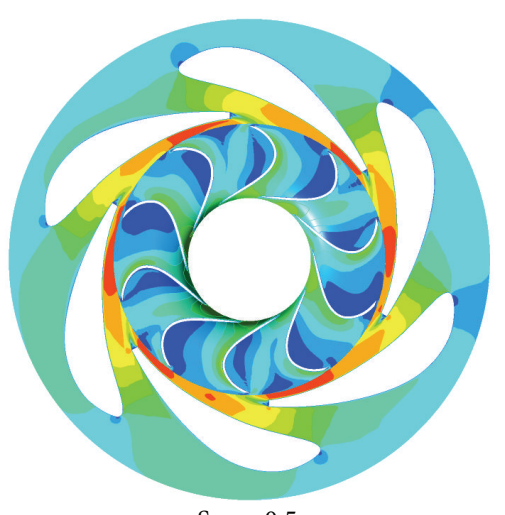

Span: 0.5

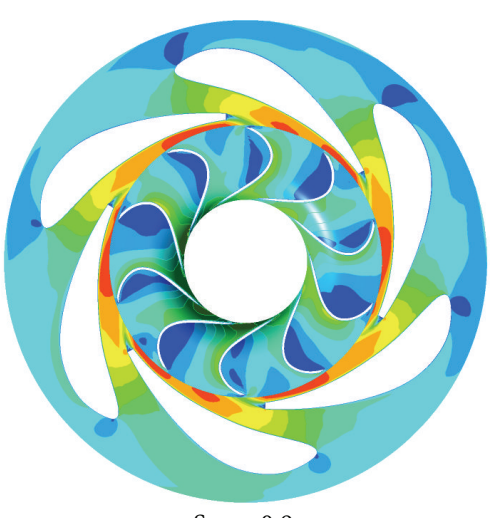

Span: 0.9

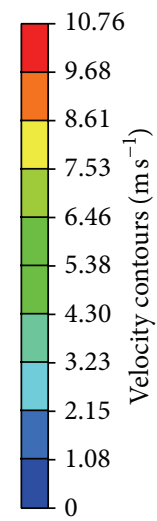

FIGURE 10: Velocity contours in distributor vanes and runner at three spans, for optimal point (BEP).

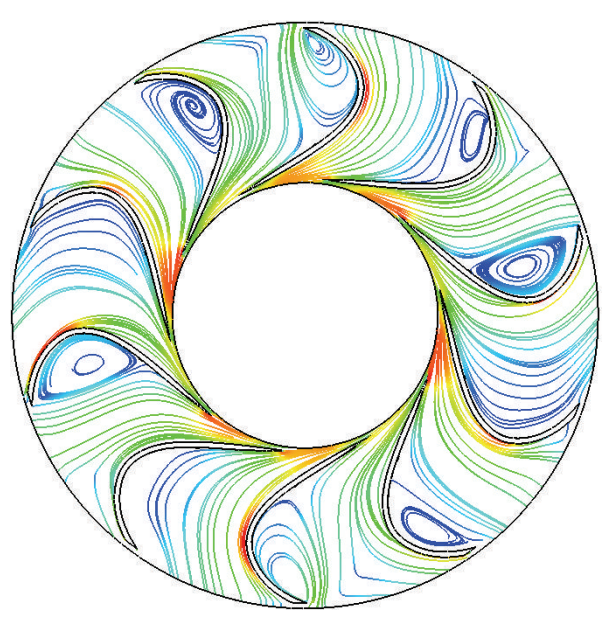

Optimal flow rate

(a)

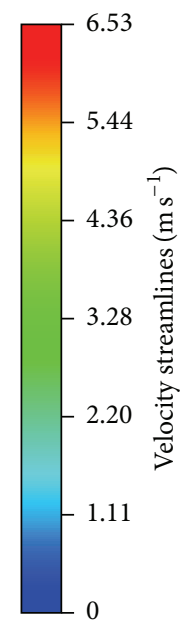

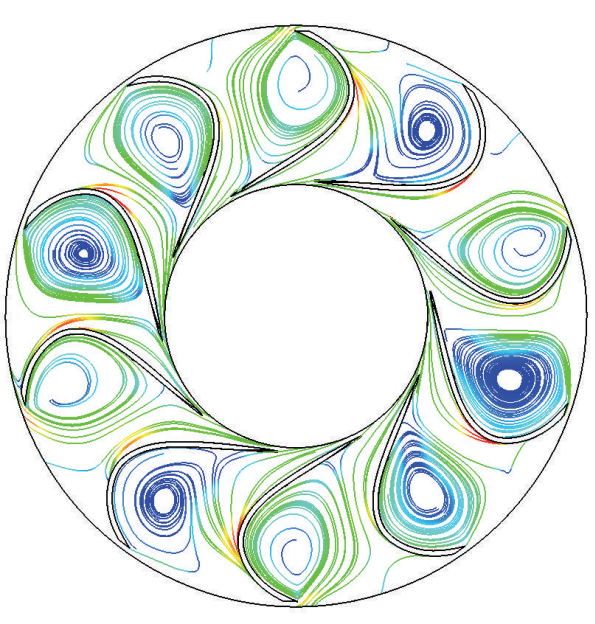

Small flow rate

(b)

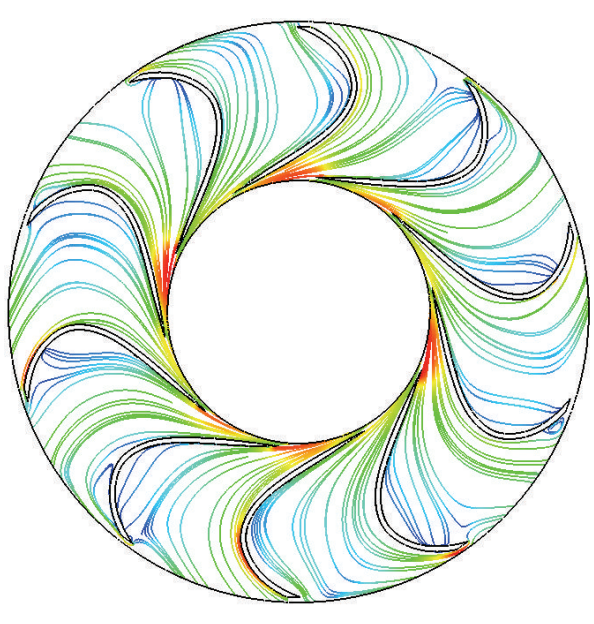

Large flow rate

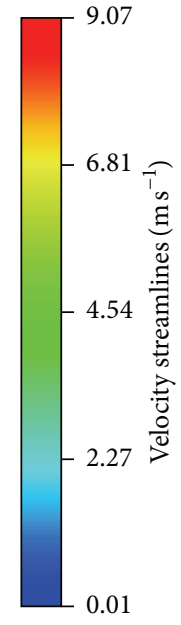

0.01
0.0

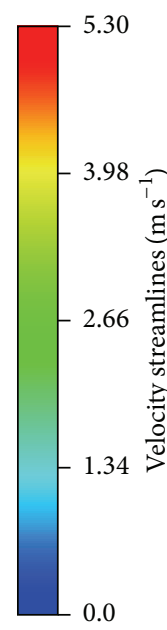

(c)

FIgURE 11: Streamlines in the runner at midspan: (a) optimal BEP, (b) low flow rate, and (c) large flow rate. 


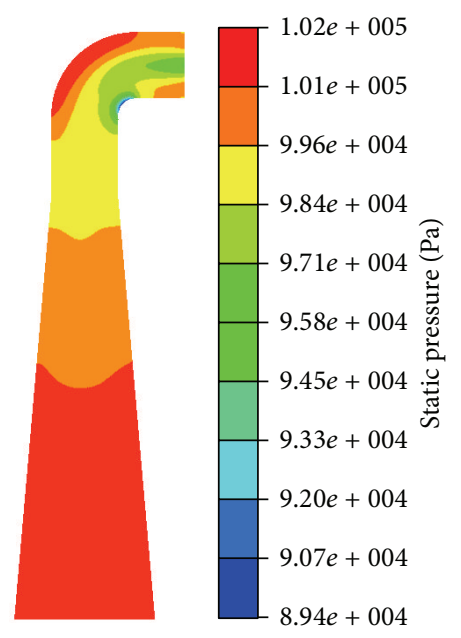

FIGURE 12: Static pressure contour in draft tube.

The swirl component of the flow velocity appearing downstream of runner at low flow rates due to the radial component of flow velocity affects greatly the flow condition in the draft tube, so an unsymmetrical and nonuniform flow is obtained with an appearance of a vortex core. This decelerated flow with a swirl results in a vortex breakdown which is considered as the main reason for a severe pressure fluctuation. Francis turbines with a fixed pitch runner have a high level of residual swirl at the inlet of draft tube due to the mismatch between the swirl generated by the distributor vanes and due to angular momentum extracted by the runner [36]. Figure 13 illustrates the velocity streamlines in the draft tube for different operating conditions, showing the effect of the elbow and tube divergence indicated by regions of high vortices at small and large flow rates. As revealed, at the optimal conditions the flow is accelerated near the inner wall of draft tube with no vortex formation. However, at small flow rate even the streamlines along the inner wall are uniform; the flow towards the outer wall is disturbed with a very large recirculation zone characterized by a jet and an eddy structure resulting in a genesis of vortex shedding. At a large flow rate, a small eddy is seen towards the exit of duct. The operation of hydraulic turbines in some off-design conditions is accompanied by a local pressure pulsation caused by rotor/stator interactions and a draft tube vortex precession propagating along the whole water conduit [37].

The performance of the draft tube is quantified by the pressure recovery coefficient $\chi=\left(P_{\text {out }}-P_{\text {in }}\right) /\left(\rho V_{\text {in }}^{2} / 2\right)$, as the ratio of the differential pressure between the inlet and outlet of draft tube and the dynamic pressure head. Figure 14 presents the actual pressure recovery coefficient for different flow coefficients, which is consistent with the experiments carried out by Tridon et al. [31]. As noticed, the best pressure recovery occurs for the optimal BEP of flow rate of $165 \mathrm{l} / \mathrm{min}$, which means that this draft tube was designed to match the point of maximum hydraulic efficiency.

3.3. Effect of Cavitation. Cavitation plays an important role in reaction water turbines such as Kaplan and Francis turbines, but the main difference between them is the design of the runner which has a clear influence on the cavitation phenomenon and its location. The other two important parameters influencing its inception and development are the machine setting level and the operation at off-design conditions, when a liquid reaches a state at which vapor cavities are formed and grow due to dynamic pressure reduction to the liquid pressure vapor. In a flowing liquid, these cavities are subjected to pressure increase that reverses their growth, collapsing implosively and disappearing. The violent process of cavity collapse takes place in a very short time of about several nanoseconds, resulting in an emission of large amplitude shock waves as demonstrated by Avellan and Farhat [38]. The tendency for a flow to cavitation is characterized by the Thoma number or plant cavitation number defined as [39]

$$
\sigma=\frac{H_{a}-H_{v a}-H_{s}-H_{v}}{H}
$$

where $H_{v a}$ is the vacuum head in a draft tank that indicates the pressure level at the outlet of a draft tube. $H_{a}$ is the atmospheric pressure head. $H_{s}$ is the suction head and $H$ is the head acting on a turbine. $H_{v}$ is the vapor pressure head at the operating temperature. From the tables of fluid properties [40], the vapor pressure at the temperature of $25^{\circ} \mathrm{C}$ is equal to 0.03166 bar. In the present simulations, $H$ is a head corresponding to $12 \mathrm{~m}$ equivalent to a pressure of 1.1772 bar and $H_{v a}+H_{s}$ is set to be equal to the static pressure at the exit of draft tube, where $H_{a}$ is equivalent to atmospheric pressure. Cavitation is treated separately from the thermal phase change as the cavitation process is typically too rapid for the assumption of thermal equilibrium at the interface to be correct. In the present cavitating flow simulation, the following assumptions are adopted from the work of Liu et al. [12]: bubble radius: $0.5 \times 10^{-6} \mathrm{~m}$, isothermal temperature equal to $198 \mathrm{~K}$, and nuclei volume fraction: $0.5 \times 10^{-5}$.

3.3.1. Cavitation Revelation. At small flow rate, the formation of cavitation pockets is near the trailing edge of the runner blade. Figure 15 presents the water vapor volume fraction over the blades and as expected higher water vapor volume fraction is seen over the suction side due to lowest pressure, typically towards the trailing edge due to flow acceleration. The volume fraction of water vapor is an important feature for cavitating flow in hydraulic turbines. At the top and front and the most of rear part of blades the volume fraction is higher than the other regions; this finding is also supported by the results of Liu et al. [12] and Jošt et al. [41]. As compared with the pressure distribution, the maximum of volume fraction of water vapor is located further away along the flow direction. This characteristic reflects the mechanism of cavitating flow for which the bubbles usually appear around a body from the micron-sized nuclei in the low-pressure regions, and when they travel with the flow they implode upon finding an adverse pressure gradient. However, if pressure reduction occurs only in a much localized region, then nuclei may not be found at this location and further reduction in the pressure is possible before cavitation occurs [4]. As such bubbles are 


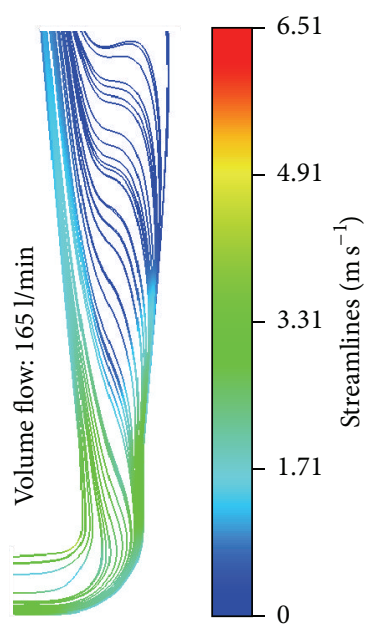

(a)

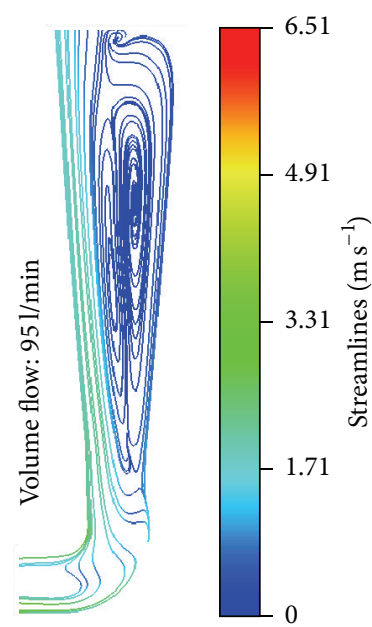

(b)

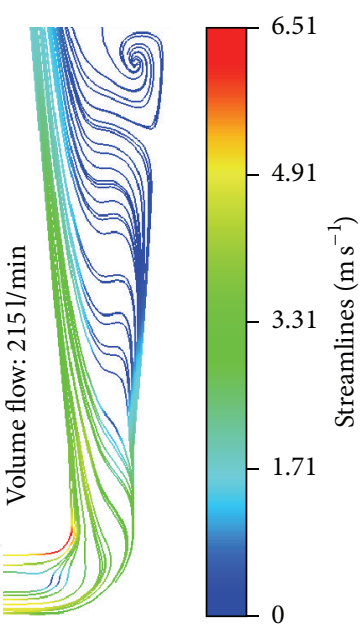

(c)

FIGURE 13: Streamlines in draft tube at midplane for different operating conditions.

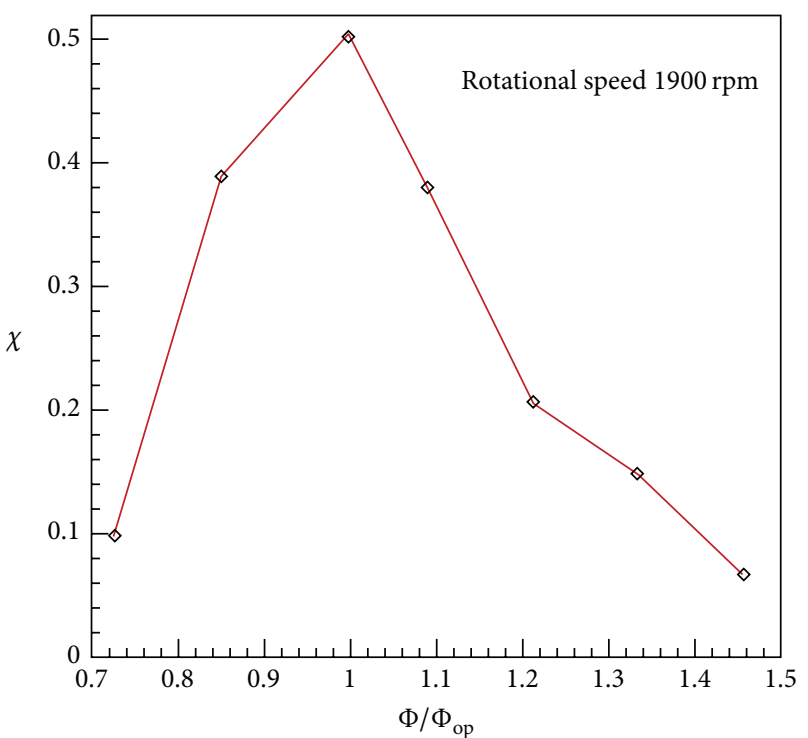

FIGURE 14: Static pressure recovery in the draft tube.

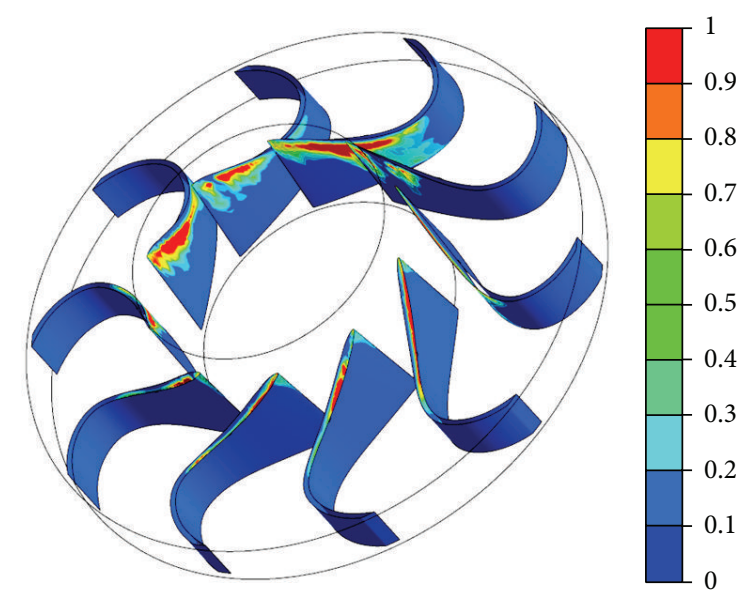

FIGURE 15: Water vapor volume fraction at the small flow rate. generated over the blading and are carried to a region where the adverse pressure gradient is higher, they implode.

If the flow from the runner has a strong swirl, the cavitated vortex rope is observed in the draft tube at a large flow rate $(215 \mathrm{l} / \mathrm{min})$. It is seen (Figure 16$)$ that the nearly straight vortex rope appears from the outlet of runner, as compared with the vortex rope [12] appearing in the inlet of draft tube at large flow rate which is less attached to the runner exit. One of the causes of such a difference may be due to the form and the geometrical dimensions of each turbine and the used cavitation number. Indeed, depending on the operating conditions, the vortex rope in the draft tube of a Francis turbine appears with different shapes [39]. Although the vortex shape is also related to the suction pressure of a turbine installation, it has been inferred that its intensity depends on the shape of the runner and the specific speed of turbine [42]. Ciocan et al. [43] observed a helical vortex rope somewhat like a twisty snake attached to the runner cone near the turbine axis and rotates around the axis of the vortex core. Towards the downstream side the vortex rope rotates with an eccentricity away from the turbine axis in the direction of the runner rotation. The difference between the present pattern of vortex rope and that in [43] is due to the steady flow simulation since unsteadiness of the vortex flow in the turbine must be large when high values of cavitation number are considered.

(a) Produced Power. The predicted power with and without cavitation model at three operating points is as follows: optimal flow rate of $165 \mathrm{l} / \mathrm{min}$, large flow rate of $215 \mathrm{l} / \mathrm{min}$, and small flow rate of $95 \mathrm{l} / \mathrm{min}$, summarized in Table 2 . At optimal and large flow rates, the power is not affected too much, explaining that the cavitation number is sufficiently accepted. At the small and large flow rate, the differences between the values of efficiency are noticeable and a cavitation can take place in these conditions because at the off-design operation the flow is more sensitive. For a turbine that works with the distributor vanes, as the flow rate is smaller than 


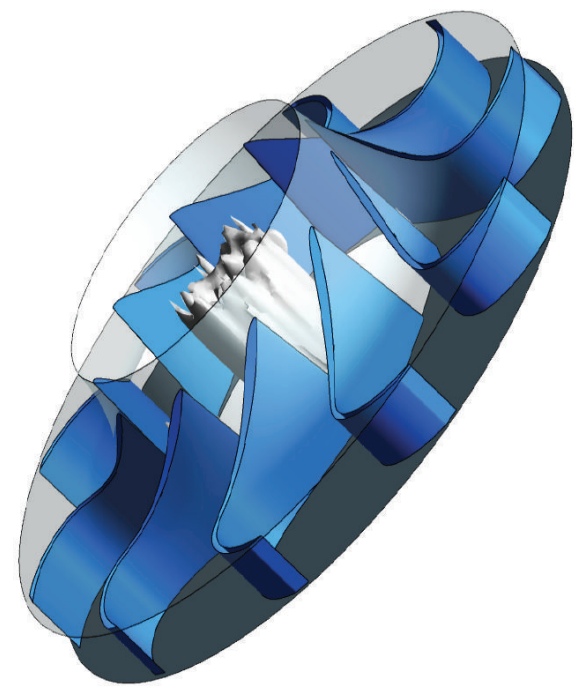

FIGURE 16: Cavitated vortex rope at large flow rate in the outlet of runner.

TABle 2: Prediction of produced power and efficiency.

\begin{tabular}{lcccc}
\hline & $\begin{array}{c}\text { Power (W) } \\
\text { without } \\
\text { cavitation }\end{array}$ & $\begin{array}{c}\text { Power (W) } \\
\text { with } \\
\text { cavitation }\end{array}$ & $\begin{array}{c}\text { Efficiency } \\
\% \\
\text { without } \\
\text { cavitation }\end{array}$ & $\begin{array}{c}\text { Efficiency } \\
\% \\
\text { with } \\
\text { cavitation }\end{array}$ \\
\hline Optimal flow rate & 189.963 & 189.970 & 79.28 & 79.28 \\
Large flow rate & 434.114 & 434.128 & 73 & 72.3 \\
Small flow rate & 98.315 & 96.553 & 72 & 70 \\
\hline
\end{tabular}

that at optimal operating regime, the runner channel is not uniformly filled as the main flow is near the shroud, and a large secondary backflow zone is formed near the hub. On the border between the backflow and the mainstream there is a strong tangential shear which is the main reason for the vortices and the appearance of the low-pressure zones that lead to a phase change which should be taken into consideration. In addition to inaccuracy attributed to the steady flow simulation the unsteadiness of the flow is large at off-design conditions. As expected for small flow rate, the fluid in the vaporous region has a little inertia in the blade relative frame, and this limits the minimum pressure on the suction side and the torque; hence the associated power decreases as the vapor region grows in size as illustrated in the work of Jošt et al. [41].

(b) Setting Level and Cavitation Number. In any hydraulic machinery, it is necessary to estimate the point at which the cavitation is expected to occur. For example, in a large axialflow pump or bulb turbine, this would be the highest point on an impeller or a runner and not the shaft centerline [44]. The determination of lower level that can be maintained at the inlet in terms of cavitation resistance is more difficult with turbines compared to pumps, because of overall cost for a turbine installation; thus a compromise must be reached in terms of achieving an acceptable power level and avoiding cavitation. This part of simulation with the cavitation
TABLE 3: Characteristics of the operating conditions.

\begin{tabular}{lccc}
\hline & $\begin{array}{c}\text { Low flow } \\
\text { rate }\end{array}$ & $\begin{array}{c}\text { Optimal } \\
\text { BEP }\end{array}$ & $\begin{array}{c}\text { Large } \\
\text { flow rate }\end{array}$ \\
\hline Efficiency (\%) & 78.87 & $79 \%$ & 78.8 \\
Produced power (W) & 112.1 & 189.96 & 347 \\
Rotational speed (rpm) & 1500 & 1900 & 2360 \\
Unit discharge $\left(\mathrm{m}^{3} / \mathrm{s}\right)$ & 0.12 & 0.14 & 0.17 \\
Unit speed $(\mathrm{rpm})$ & 37.95 & 48.06 & 59.7 \\
Unit power $(\mathrm{W})$ & 413.46 & 710.4 & 1305 \\
\hline
\end{tabular}

TABLE 4: Cavitation number and boundaries conditions.

\begin{tabular}{lccc}
\hline $\begin{array}{l}\text { Simulations for } \\
\text { operating points }\end{array}$ & $\begin{array}{c}\text { Cavitation } \\
\text { number }\end{array}$ & $\begin{array}{c}\text { Inlet } \\
\text { head (m) }\end{array}$ & $\begin{array}{c}\text { Static } \\
\text { pressure at } \\
\text { outlet (bar) }\end{array}$ \\
\hline 1 & 0.08 & 12 & 0.89 \\
2 & 0.11 & - & 0.85 \\
3 & 0.15 & - & 0.8 \\
4 & 0.20 & - & 0.74 \\
5 & 0.25 & - & 0.68 \\
6 & 0.33 & - & 0.59 \\
7 & 0.40 & - & 0.51 \\
8 & 0.48 & - & 0.41 \\
\hline
\end{tabular}

model consists of determining the setting level in order to overcome any hydraulic efficiency alteration and minimizing the erosion risk. Each type of cavitation is considered with respect to its dependence on the value of setting level. The onset of blade leading edge cavity is more influenced by the blade geometry and the flow incidence compared to the value of cavitation number $\sigma$. On the other hand, the cavity development corresponding to the design operating point such as bubble cavitation as well as hub cavity is very sensitive to the Thoma number. As different types of cavitation arise depending either on the blade design and operating point or on the Thoma number, it is important to examine the type of cavitation occurring in the operating range.

The setting level of a machine determines the pressure field in relation to the vapor pressure threshold. The bubble cavitation can appear even at the best efficiency operating point because it has a strong dependence on this level. To analyze the influence of cavitation number and the setting level on the hydraulic efficiency and power, simulations for three different operating points (Table 3 ) which corresponds to the results obtained without cavitation, which corresponds to the maximum of efficiency for three different rotational speeds.

For each operating point, eight simulations were performed at different values of cavitation number defined as follows: $\sigma_{0}$ is lowest value for which the efficiency remains unchanged and $\sigma_{P}$ the is safety margin for plant. The boundary conditions applied to achieve these eight values of cavitation number are the hydraulic head in terms of total pressure at the inlet of spiral casing that remains constant for all the simulations, whereas the eight different values of setting level are adjusted in the boundary conditions as a static pressure at outlet (Table 4 ). The three cases corresponding to operating conditions at low flow rate, optimal BEP, 


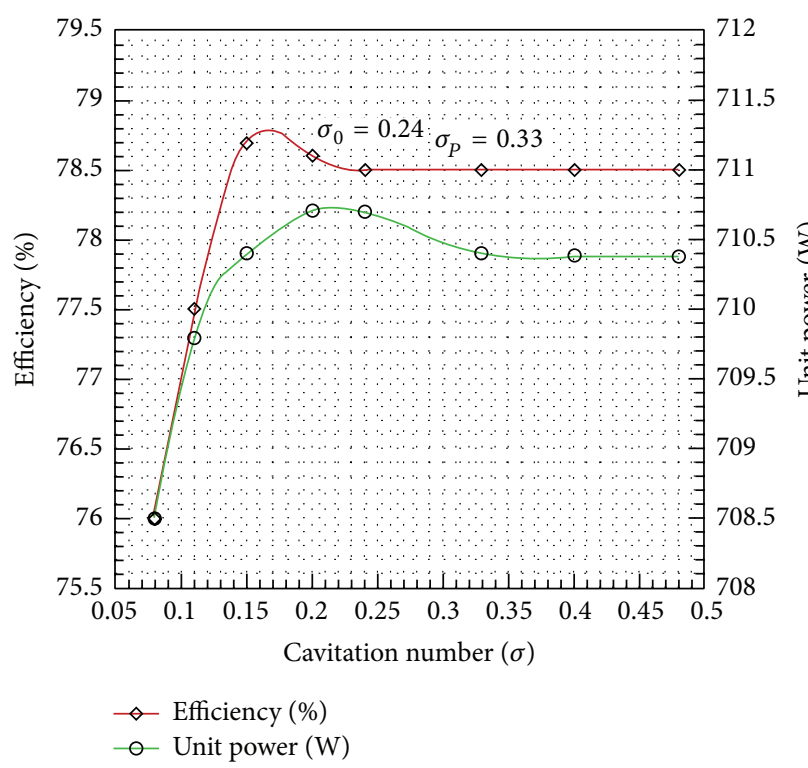

(a)

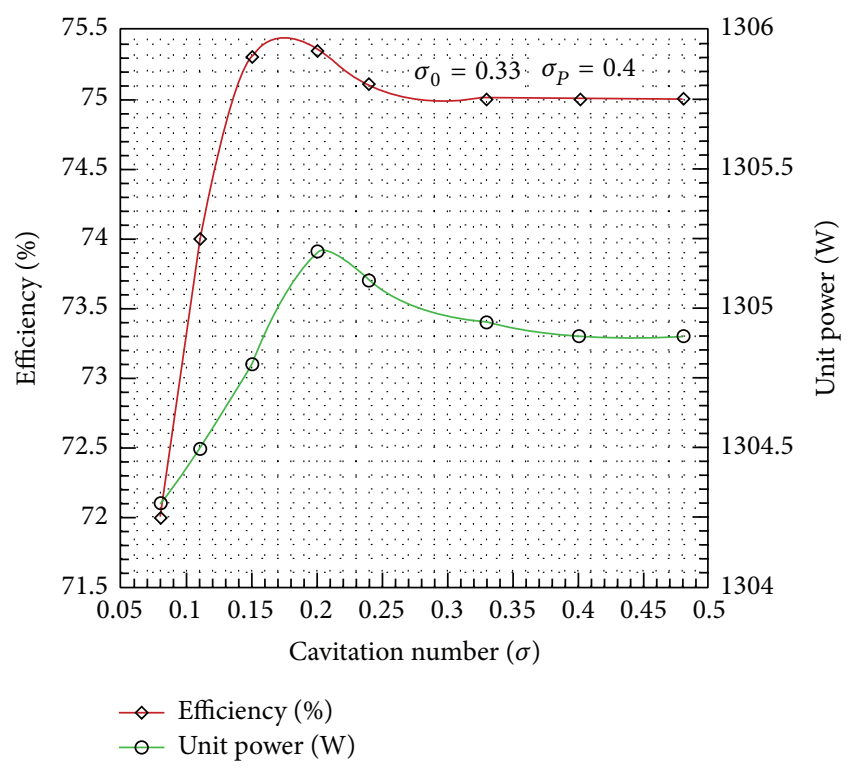

(b)

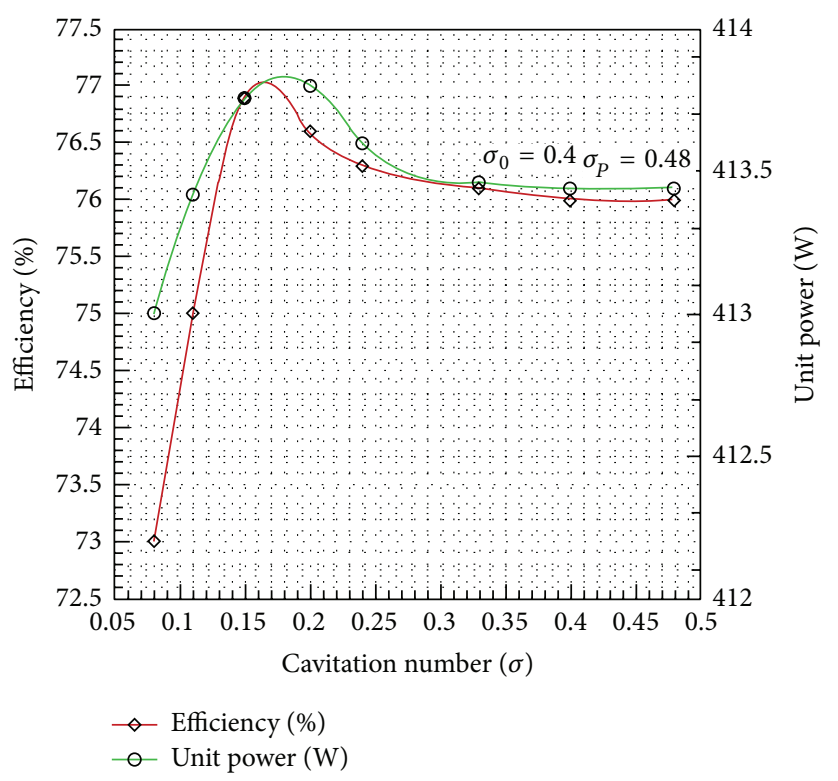

(c)

Figure 17: Cavitation effects on hydraulic efficiency and unit power: (a) at optimal point (BEP), (b) at large flow rate, and (c) at small flow rate.

and large flow rate are set by the distributor vane opening. Therefore, this primary method for the cavitation detection consists in gradually reducing the suction head (exit pressure from draft tube) under constant operating conditions, and the changes in the produced head and power and efficiency can be attributed directly to the cavitation phenomena.

Figure 17 presents the cavitating characteristics for the optimal BEP, large flow rate, and low flow rate, showing that the predicted hydraulic efficiency for all operations points is less than that without cavitation. There are noticeable differences in hydraulic efficiency for optimal BEP, large flow rate, and low flow rate for, respectively, 3.62\%, 4.86\%, and 5.45\%, but for the unit power it is only $0.31 \%, 0.076 \%$, and $0.48 \%$, respectively, presenting more stability than the efficiency. The curves show that at some limiting cavitation number the curve of performance becomes nearly vertical and the drop becomes severe at the breakdown point. It is interesting to note that this point (bend) of the curve corresponds roughly to the development of a cavitation zone over the length of blade. Also, a plateau in the performance variation at higher values of cavitation number is observed. In addition the value of cavitation number for the optimal BEP is smaller than that at large or small flow rate, which may be explained by the smaller cavitation number compared to that at off-design operating conditions. It should be taken into account that the unit power is more dependent upon the rotational speed 
and the torque produced by the runner without taking into consideration the flow behavior in the draft tube where many different types of cavitation could appear. For this reason and in order to calculate the unit power with cavitation model it is important to predict the flow behavior in the runner, but it is not a sufficient indicator as the efficiency which is a good indicator. As a consequence, the setting level of the Francis turbine is determined according to the risk of efficiency alteration which is higher for the large discharge operating conditions, as it can be seen from the expression of cavitation number where $\sigma_{P}$ is predicted with the value greater than 0.4 which corresponds to the safety margin.

\section{Conclusion}

The role of each component of this horizontal small Francis turbine and its effect on the hydrodynamic performance were investigated by simulating the single phase and the cavitating turbulent flows considering SST turbulence model and mixture cavitation model over the entire flow passages. The predicted performance depicts that the nominal point corresponds to a maximum of hydraulic efficiency of $79.28 \%$ and an important drop with increased discharge. The details of flow structures show that the most of losses are located in the runner where there are large vortices affecting the stability of operation. The velocity at inlet of draft tube has a substantial circumferential component that initiates a precession motion of a vortex of helical shape. According to the results without cavitation, it is clear that the off-design operating points are more affected, and from the simulations performed at different values of cavitation number the following conclusions are drawn:

(i) The hydraulic efficiency is more affected by cavitation number compared to unit power.

(ii) The loss of hydraulic efficiency is shown to increase with the suction head.

(iii) The value of cavitation number for the optimal point (BEP) is smaller than that at off-design.

(iv) The cavitation number for the safety margin for a plant is equal to 0.48 .

\section{Nomenclature}

$\Phi:$ Discharge coefficient

$\psi$ : Energy coefficient

Q: Flow rate

$D$ : External diameter of runner

$\omega$ : Angular velocity

$\Omega$ : Vector of angular velocity

$H$ : Head, total enthalpy

$h$ : Static enthalpy

$N$ : Rotational speed

$\eta$ : Efficiency

$\chi$ : Recovery coefficient
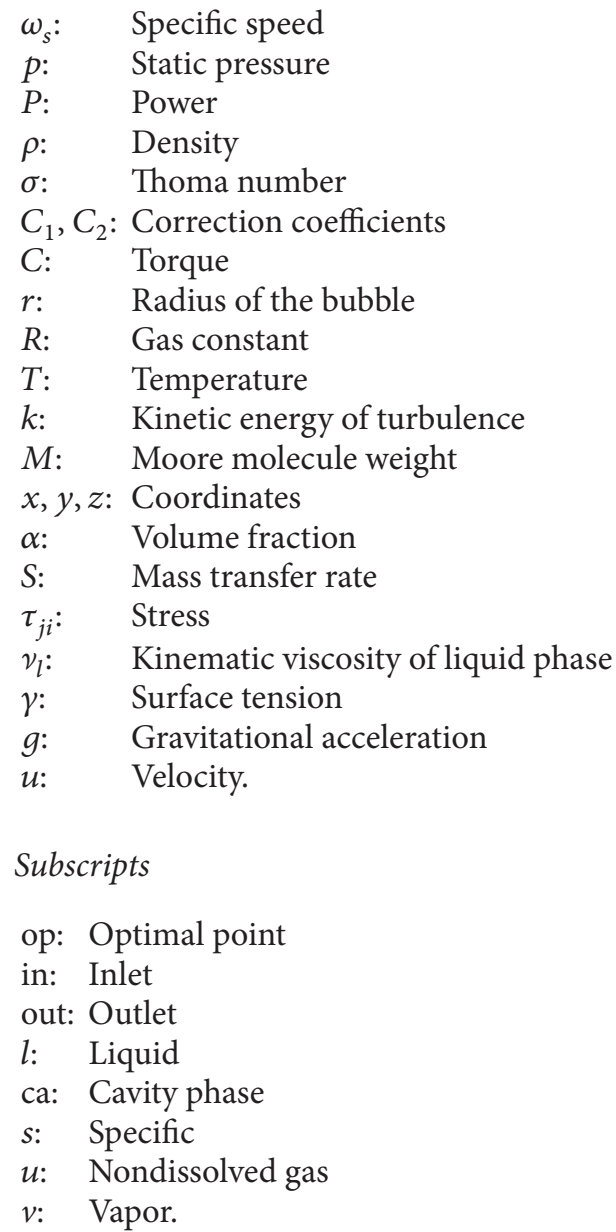

\section{Competing Interests}

The authors declare no competing interests.

\section{References}

[1] K. C. Anup, B. Thapa, and Y.-H. Lee, "Transient numerical analysis of rotor-stator interaction in a Francis turbine," Renewable Energy, vol. 65, pp. 227-235, 2014.

[2] S. Romeo, M. Sebastian, A. Ioan, and B. Sandor, "Numerical investigation of 3D cavitating flow in Francis turbines," in Proceedings of the 12th International Conference on modelling Fluid Flow Technologies (CMFF '03), Budapest, Hungary, September 2003.

[3] Multiphase Flow Theory, ANSYS CFX-Solver Theory Guide, 2015.

[4] C. E. Brennen, Cavitation and Bubble Dynamics, Oxford University Press, 1995.

[5] K. Helmut, D. Peter, and S. Mirjam, "Numerical hill chart prediction by means of CFD stage simulation for a complete Francis turbine," in Proceedings of the 18th IAHR Symposium on Hydraulic Machinery and Cavitation, Valencia, Spain, 1996.

[6] S. Mirjam, V. C. Michael, and F. G. Paul, "Validation of a stage calculation in a Francis turbine," in Proceedings of the 18th IAHR Symposium on Hydraulic Machinery and Cavitation, Valencia, Spain, 1996.

[7] M. Sabourin, Y. Labrecque, and V. De Henau, "From components to complete turbine numerical simulation," in Proceedings 
of the 18th IAHR Symposium on Hydraulic Machinery and Cavitation, Valencia, Spain, 1996.

[8] J. Wu, K. Shimmei, K. Tani, K. Niikura, and J. Sato, "CFDbased design optimization for hydro turbines," Journal of Fluids Engineering, vol. 129, no. 2, pp. 159-168, 2007.

[9] P. Kumar and R. P. Saini, "Study of cavitation in hydro turbines-a review," Renewable and Sustainable Energy Reviews, vol. 14, no. 1, pp. 374-383, 2010.

[10] I. Senocak and W. Shyy, "A pressure-based method for turbulent cavitating flow computations," Journal of Computational Physics, vol. 176, no. 2, pp. 363-383, 2002.

[11] Y. Wu, S. Liu, H.-S. Dou, and L. Zhang, "Simulations of unsteady cavitating turbulent flow in a Francis turbine using the RANS method and the improved mixture model of two-phase flows," Engineering with Computers, vol. 27, no. 3, pp. 235-250, 2011.

[12] S. Liu, L. Zhan, M. Nishi, and Y. Wu, "Cavitating turbulent flow simulation in a Francis turbine based on mixture model," Journal of Fluids Engineering, vol. 131, no. 5, Article ID 051302, 2009.

[13] S. Kurosawa, M. S. Lim, and Y. Enomoto, "Virtual model test for a Francis turbine," in Proceedings of the 25th IAHR Symposium on Hydraulic Machinery and Systems, 2010.

[14] Y. Wu, J. Liu, Y. Sun, S. Liu, and Z. Zuo, "Numerical analysis of flow in a Francis turbine on an equal critical cavitation coefficient line," Journal of Mechanical Science and Technology, vol. 27, no. 6, pp. 1635-1641, 2013.

[15] Z. Yaping, L. Weili, R. Hui, and L. Xingqi, "Performance study for Francis-99 by using different turbulence models," Journal of Physics: Conference Series, vol. 579, no. 1, Article ID 012012, 2015.

[16] J. Yang, L. J. Zhou, and Z. W. Wang, “The numerical research of runner cavitation effects on spiral vortex rope in draft tube of Francis turbine," Journal of Physics: Conference Series, vol. 656, no. 1, Article ID 012076, 2015.

[17] J. Wack and S. Riedelbauch, "Numerical simulations of the cavitation phenomena in a Francis turbine at deep part load conditions," Journal of Physics: Conference Series, vol. 656, no. 1, Article ID 012074, 2015.

[18] G. Mousmoulis, I. Kassanos, J. Anagnostopoulos, and D. Papantonis, "Experimental and numerical investigation ofthe cavitating draft tube vortex in a Francis model turbine," in Proceedings of the 6th IAHR International Meeting of the Workgroup on Cavitation and Dynamic Problems in Hydraulic Machinery and Systems, Ljubljana, Slovenia, September 2015.

[19] P. P. Gohil and R. P. Saini, "Effect of temperature, suction head and flow velocity on cavitation in a Francis turbine of small hydro power plant," Energy, vol. 93, pp. 613-624, 2015.

[20] J. Decaix, A. Müller, F. Avellan, and C. Münch, "RANS computations of a cavitating vortex rope at full load," in Proceedings of the 6th IAHR Meeting of the Working Group (IAHRWG '15), Ljubljana, Slovenia, September 2015.

[21] I. Kassanos, J. Anagnostopoulos, and D. Papantonis, "Numerical analysis of the effect of splitter blades on draft tube cavitation of a low specific speed Francis turbine," in Proceedings of the 6th IAHR Meeting of the Working Group (IAHRWG '15), Ljubljana, Slovenia, September 2015.

[22] P. P. Gohil and R. P. Saini, "Numerical study of cavitation in Francis turbine of a small hydro power plant," Journal of Applied Fluid Mechanics, vol. 9, no. 1, pp. 357-365, 2016.

[23] F. R. Menter, "Two-equation eddy-viscosity turbulence models for engineering applications," AIAA Journal, vol. 32, no. 8, pp. 1598-1605, 1994.
[24] C. Trivedi, M. J. Cervantes, B. K. Gandhi, and O. G. Dahlhaug, "Experimental and numerical studies for a high head Francis turbine at several operating points," Journal of Fluids Engineering, vol. 135, no. 11, Article ID 111102, 17 pages, 2013.

[25] C. Widmer, T. Staubli, and N. Ledergerber, "Unstable characteristics and rotating stall in turbine brake operation of pumpturbines," Journal of Fluids Engineering, vol. 133, no. 4, Article ID 041101, 9 pages, 2011.

[26] International Electrotechnical Commission (IEC), Field Acceptance Tests to Determine the Hydraulic Performance of Hydraulic Turbines, Storage Pumps and Pump-Turbines, IEC 60041, International Electrotechnical Commission (IEC), Geneva, Switzerland, 3rd edition, 1991.

[27] ANSYS, ANSYS CFX-Release 12.0, ANSYS, Canonsburg, Pa, USA, 2009.

[28] K. Okita and T. Kajishima, "Numerical simulation of unsteady cavitating flows around a hydrofoil," Transactions of the Japan Society of Mechanical Engineers Part B, vol. 68, no. 667, pp. 637644, 2002.

[29] A. K. Singhal, N. Vaidya, and A. D. Leonard, "Multidimensional simulation of cavitating flows using a PDF model for phase change," in Proceedings of the ASME Fluids Engineering Division Summer Meeting, vol. 4, pp. 1-8, Vancouver, Canada, 1997.

[30] H. K. Cammenga, "Evaporation mechanisms of liquids," Current Topics in Materials Science, vol. 5, pp. 335-446, 1980.

[31] S. Tridon, S. Barre, G. D. Ciocan, and L. Tomas, "Experimental analysis of the swirling flow in a Francis turbine draft tube: focus on radial velocity component determination," European Journal of Mechanics, B/Fluids, vol. 29, no. 4, pp. 321-335, 2010.

[32] G. A. Aggidis and A. Židonis, "Hydro turbine prototype testing and generation of performance curves: fully automated approach," Renewable Energy, vol. 71, pp. 433-441, 2014.

[33] J. Leonard Daniel and W. Lindau Jules, "Multiphase computation of cavitation breakdown in model and prototype scale Francis turbines," in Proceedings of the ASME-JSME-KSME Joint Fluids Engineering Conference, Seoul, South Korea, July 2015.

[34] J. G. Hellström, B. D. Marjavaara, and T. S. Lundström, "Parallel CFD simulations of an original and redesigned hydraulic turbine draft tube," Advances in Engineering Software, vol. 38, no. 5, pp. 338-344, 2007.

[35] H. Xiao and B. Yu, "3D-viscous flow simulation and performance prediction of a complete model francis turbine," in Proceedings of the International Conference on Mechanic Automation and Control Engineering (MACE '10), pp. 39423944, IEEE, Wuhan, China, June 2010.

[36] S. R. Romeo and M. Sebastian, "Decelerated swirling flow control in the discharge cone of francis turbines," in Fluid Machinery and Fluid Mechanics: 4th International Symposium (4th ISFMFE), pp. 89-96, Springer, Berlin, Germany, 2009.

[37] D. Chirkov, A. Avdyushenko, L. Panov, D. Bannikov, S. Cherny, and V. Skorospelov, "CFD simulation of pressure and discharge surge in Francis turbine at off-design conditions," in Proceedings of the 26th IAHR Symposium on Hydraulic Machinery and Systems, Beijing, China, August 2012.

[38] F. Avellan and M. Farhat, "Shock pressure generated by cavitation vortex collapse," in Proceedings of 3rd International Symposium on Cavitation Noise and Erosion in Fluid System of the ASME Winter Annual Meeting, FED, vol. 88, pp. 119-125, San Francisco, Calif, USA, December 1989.

[39] C. H. Arn, P. H. Dupont, and F. Avellan, "Efficiency alteration of Francis turbines by travelling bubble cavitation," in Proceedings 
of the 8th IAHR Symposium Hydraulic Machinery and Cavitation, Valencia, Spain, September 1996.

[40] G. F. C. Rogers and Y. R. Mayhew, Thermodynamic and Transport Properties of Fluids, Blackwell, Malden, Mass, USA, 5th edition, 1995.

[41] D. Jošt, M. Morgut, A. Škerlavaj, and E. Nobile, "Cavitation prediction in a Kaplan turbine using standard and optimized model parameters," in Proceedings of the 6th IAHR Meeting of the Working Group on Cavitation and Dynamic Problems in Hydraulic Machinery and Systems, Ljubljana, Slovenia, September 2015.

[42] A. Ruprecht, T. Helmrich, T. Aschenbrenner, and T. Scherer, "Simulation of vortex rope in a turbine draft tube," in Proceedings of the 21st IAHR Symposium on Hydraulic Machinery and Systems, Lausanne, Switzerland, September 2002.

[43] G. D. Ciocan, M. S. Iliescu, T. C. Vu, B. Nennemann, and F. Avellan, "Experimental study and numerical simulation of the FLINDT draft tube rotating vortex," Journal of Fluids Engineering, vol. 129, no. 2, pp. 146-158, 2007.

[44] T. Tanaka, "Thermodynamic effect and cavitation performance of a cavitating centrifugal pump," in Proceedings of the ASMEJSME-KSME Joint Fluids Engineering Conference, Hamamatsu, Japan, July 2011. 


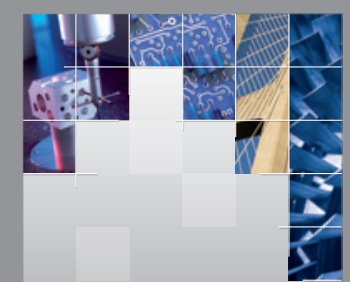

\section{Enfincering}
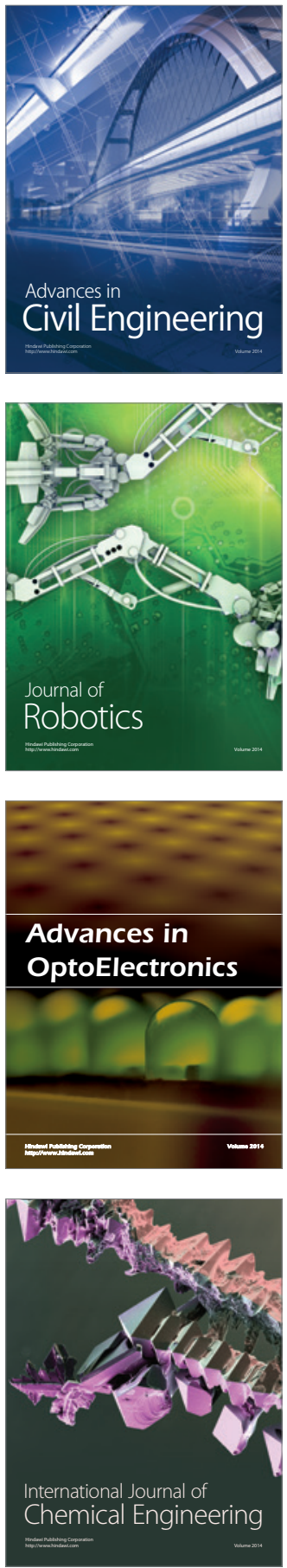

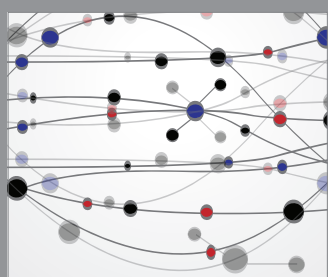

The Scientific World Journal

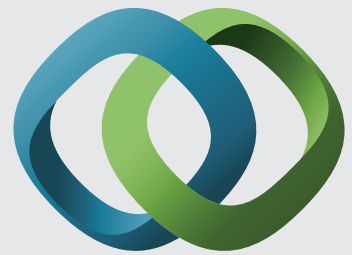

\section{Hindawi}

Submit your manuscripts at

http://www.hindawi.com
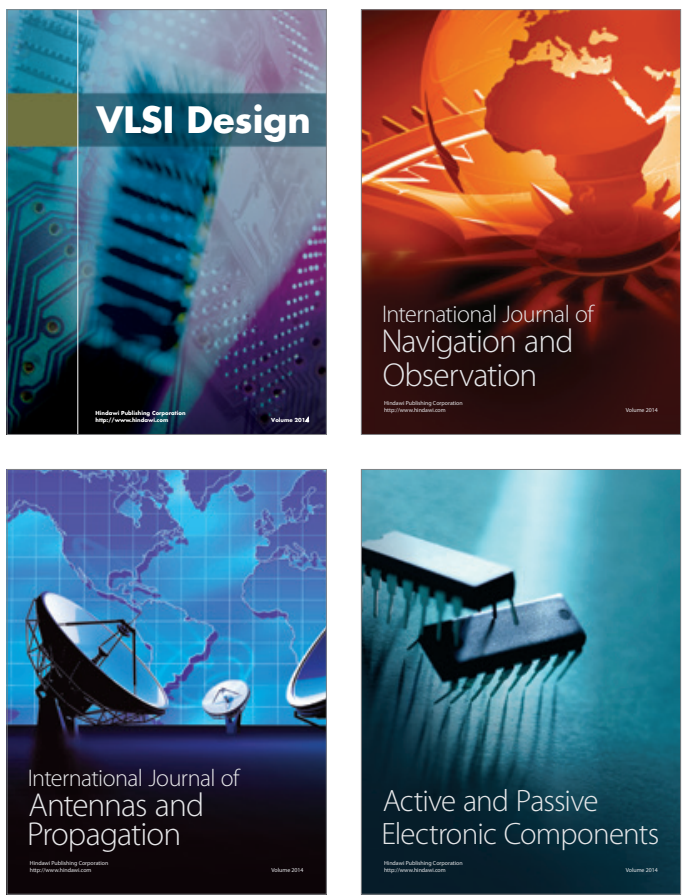
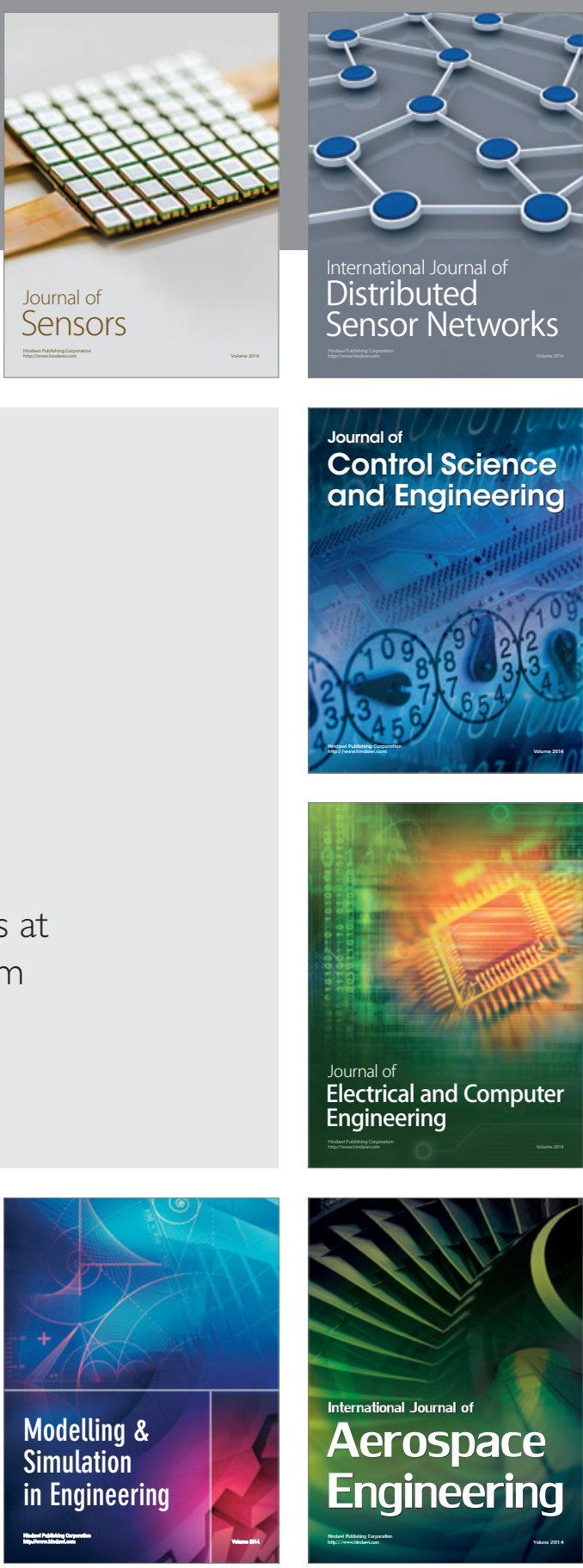

International Journal of

Distributed

Sensor Networks

Journal of

Control Science

and Engineering
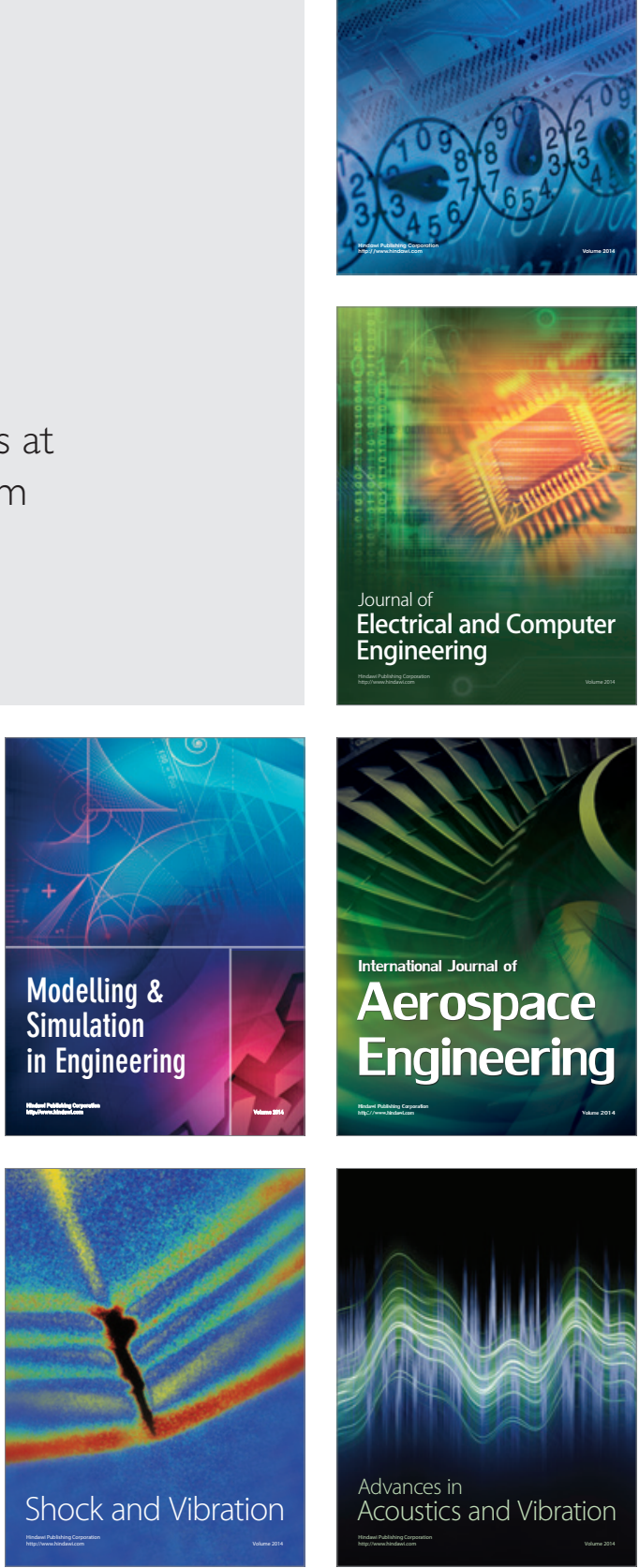